1 Hacettepe Journal of Mathematics and Statistics

$\bigcap$ Volume $44(5)$ (2015), $1011-1022$

\title{
Simplicial homology groups of certain digital surfaces
}

\author{
Emel ÜNVER DEMIR * *and İsmet KARACA丹
}

\begin{abstract}
In this paper we compute the simplicial homology groups of some digital surfaces.
\end{abstract}

Keywords: Digital topology, digital surface, simplicial homology groups.

2000 AMS Classification: 55N35, 68R10, 68U05, 68U10.

Received 26/03/2012 : Accepted 13/06/2014 Doi : 10.15672/HJMS.2015449657

\section{INTRODUCTION}

Digital topology [19, 17] has been used in different image processing and computer graphics algorithms for several decades. It addresses the fundamental properties of binary object connectivity in two dimensional (2D) and three dimensional (3D) digital images. Concepts and results of Digital Topology are used to specify and justify some important low-level image processing algorithms including algorithms for thinning, boundary extraction, object counting, and contour filling. The properties of digital images with tools from Topology (including Algebraic Topology) are used by many researchers $[1-12,16,17,19]$.

Homology is a powerful topological invariant which characterizes an object by its $p$-dimensional holes. Intuitively the 0-dimensional holes can be seen as "tiny holes", 1-dimensional holes can be seen as tunnels, and 2-dimensional holes can be seen as cavities. The usage of homology groups is a new topic and is not widely spread. Simplicial homology groups of digital images have been studied by several researchers [1, 10, 16]. Boxer et al. [10] extend results of [1] about computing simplicial homology groups of digital images. In this work, we compute simplicial homology groups of certain minimal simple closed surfaces.

This paper is organized as follows. Section 2 provides some basic notions used in this paper. In section 3 , we compute the simplicial homology groups of certain digital surfaces.

*Department of Mathematics, Celal Bayar University, Muradiye Campus, Manisa, Turkey Email: emel.unver@cbu.edu.tr

${ }^{\dagger}$ Department of Mathematics, Ege University, Bornova, Izmir 35100, TURKEY.

Email:ismet.karaca@ege.edu.tr 


\section{PRELIMINARIES}

Let $\mathbb{Z}^{n}$ be the set of lattice points in the $n$-dimensional Euclidean space where $\mathbb{Z}$ is the set of integers. For a positive integer $l$ with $1 \leq l \leq n$ and two distinct points $p=\left(p_{1}, p_{2}, \ldots, p_{n}\right), q=\left(q_{1}, q_{2}, \ldots, q_{n}\right) \in \mathbb{Z}^{n}, p$ and $q$ are $c_{l}$-adjacent [8] if

(1) there are at most $l$ indices $i$ such that $\left|p_{i}-q_{i}\right|=1$; and

(2) for all other indices $i$ such that $\left|p_{i}-q_{i}\right| \neq 1, p_{i}=q_{i}$.

Another commonly used notation for $c_{l}$-adjacency reflects the number of neighbors $q \in \mathbb{Z}^{n}$ that a given point $p \in \mathbb{Z}^{n}$ may have under the adjacency. For example, if $n=1$ we have $c_{1}=2$-adjacency; if $n=2$ we have $c_{1}=4$-adjacency and $c_{2}=8$-adjacency; if $n=3$ we have $c_{1}=6$-adjacency, $c_{2}=18$-adjacency, and $c_{3}=26$-adjacency [8]. Given a natural number $l$ in conditions (1) and (2) with $1 \leq l \leq n, l$ determines each of the $\kappa$-adjacency relations of $\mathbb{Z}^{n}$ in terms of (1) and (2) [14] as follows.

$$
\kappa \in\left\{2 n(n \geq 1), 3^{n}-1(n \geq 2), 3^{n}-\sum_{t=0}^{r-2} C_{t}^{n} 2^{n-t}-1(2 \leq r \leq n-1, n \geq 3)\right\}
$$

The pair $(X, \kappa)$ is considered in a digital picture $\left(\mathbb{Z}^{n}, \kappa, \bar{\kappa}, X\right)$ for $n \geq 1$ in $[3,4,6,13$, which is called a digital image where $(\kappa, \bar{\kappa}) \in\left\{(\kappa, 2 n),\left(2 n, 3^{n}-1\right)\right\}$. Each of $\kappa$ and $\bar{\kappa}$ is one of the general $\kappa$-adjacency relations. We usually do not permit that $\kappa$ and $\bar{\kappa}$ both equal $2 n$ when $n>1$, because of the digital connectivity paradox 18 . For instance, $(\kappa, \bar{\kappa}) \in\{(4,8),(8,4)\}$ and $\{(6,18),(6,26),(26,6),(18,6)\}$ are usually considered in $\mathbb{Z}^{2}$ and $\mathbb{Z}^{3}$, respectively [6, 13, 19, 20].

A digital interval is a set of the form $[a, b]_{\mathbb{Z}}=\{z \in \mathbb{Z} \mid a \leq z \leq b\}$ where $a, b \in \mathbb{Z}$ with $a<b$.

Let $\kappa$ be an adjacency relation on $\mathbb{Z}^{n}$. A $\kappa$-neighbor of a lattice point $p$ is $\kappa$-adjacent to $p$. A digital image $X \subset \mathbb{Z}^{n}$ is $\kappa$-connected [15] if and only if for every pair of different points $x, y \in X$, there is a set $\left\{x_{0}, x_{1}, \ldots, x_{r}\right\}$ of points of a digital image $X$ such that $x=x_{0}, y=x_{r}$ and $x_{i}$ and $x_{i+1}$ are $\kappa$-neighbors where $i=0,1, \ldots, r-1$. A $\kappa$-component of a digital image $X$ is a maximal $\kappa$-connected subset of $X$.

Let $X \subset \mathbb{Z}^{n_{0}}$ and $Y \subset \mathbb{Z}^{n_{1}}$ be digital images with $\kappa_{0}$ and $\kappa_{1}$-adjacency respectively. Then the function $f: X \rightarrow Y$ is called $\left(\kappa_{0}, \kappa_{1}\right)$-continuous [6] 20] if for every $\kappa_{0}$-connected subset $U$ of $X, f(U)$ is a $\kappa_{1}$-connected subset of $Y$. We say that such a function is digitally continuous. Similar notions are defined on discrete manifolds in [11]: Let $D_{1}$ and $D_{2}$ be two discrete manifolds and $f: D_{1} \rightarrow D_{2}$ be a mapping. $f$ is said to be an immersion from $D_{1}$ to $D_{2}$ or a gradually varied operator if $x$ and $y$ are adjacent in $D_{1}$ implies either $f(x)=f(y)$ or $f(x), f(y)$ are adjacent in $D_{2}$.

Let $X$ be a digital image with $\kappa$-adjacency. If $f:[0, m]_{\mathbb{Z}} \rightarrow X$ is a $(2, \kappa)$-continuous function such that $f(0)=x$ and $f(m)=y$, then $f$ is called a digital path from $x$ to $y$ in $X$. If $f(0)=f(m)$ then the $\kappa$-path is said to be closed, and the function is called a $\kappa$-loop. Let $f:[0, m-1]_{\mathbb{Z}} \rightarrow X$ be a $(2, \kappa)$-continuous function such that $f(i)$ and $f(j)$ are $\kappa$-adjacent if and only if $j=i \pm 1 \bmod m$. Then the set $f\left([0, m-1]_{\mathbb{Z}}\right)$ is called a simple closed $\kappa$-curve. A point $x \in X$ is called a $\kappa$-corner, if $x$ is $\kappa$-adjacent to two and only two points $y, z \in X$ such that $y$ and $z$ are $\kappa$-adjacent to each other [4]. Moreover, the $\kappa$-corner $x$ is called simple if $y, z$ are not $\kappa$-corners and if $x$ is the only point $\kappa$-adjacent to both $y, z[3 . X$ is called a generalized simple closed $\kappa$-curve if what is obtained by removing all simple $\kappa$-corners of $X$ is a simple closed $\kappa$-curve [4. If $(X, \kappa)$ is a $\kappa$-connected digital image in $\mathbb{Z}^{3},|X|^{x}=N_{3}^{*}(x) \cap X$, where $N_{3}^{*}(x)=\left\{x^{\prime} \in \mathbb{Z}^{3}: x\right.$ and $x^{\prime}$ are 26-adjacent $\}$ [3, 4]. Generally, if $(X, \kappa)$ is a $\kappa$-connected digital image in $\mathbb{Z}^{n},|X|^{x}=N_{n}^{*}(x) \cap X$, where $N_{n}^{*}(x)=\left\{x^{\prime} \in \mathbb{Z}^{n}: x\right.$ and $x^{\prime}$ are $c_{n}$-adjacent $\}[13$.

Let $X \subset \mathbb{Z}^{n_{0}}$ and $Y \subset \mathbb{Z}^{n_{1}}$ be digital images with $\kappa_{0}$ and $\kappa_{1}$-adjacency respectively. A function $f: X \rightarrow Y$ is a $\left(\kappa_{0}, \kappa_{1}\right)$-isomorphism [9] (called $\left(\kappa_{0}, \kappa_{1}\right)$-homeomorphism in 
[5]) if $f$ is $\left(\kappa_{0}, \kappa_{1}\right)$-continuous, bijective and $f^{-1}: Y \rightarrow X$ is $\left(\kappa_{1}, \kappa_{0}\right)$-continuous, in which case we write $X \approx_{\left(\kappa_{0}, \kappa_{1}\right)} Y$.

2.1. Definition. [13] Let $c^{*}:=\left\{x_{0}, x_{1}, \ldots, x_{n}\right\}$ be a closed $\kappa$-curve in $\mathbb{Z}^{2}$ where $\{\kappa, \bar{\kappa}\}=$ $\{4,8\}$. A point $x$ of the complement $\overline{c^{*}}$ of a closed $\kappa$-curve $c^{*}$ in $\mathbb{Z}^{2}$ is said to be in the interior of $c^{*}$ if it belongs to the bounded $\bar{\kappa}$-connected component of $\overline{c^{*}}$. The set of all interior points of $c^{*}$ is denoted by $\operatorname{Int}\left(c^{*}\right)$.

2.2. Definition. [13] Let $(X, \kappa)$ be a digital image in $\mathbb{Z}^{n}, n \geq 3$ and $\bar{X}=\mathbb{Z}^{n}-X$. Then $X$ is called a closed $\kappa$-surface if it satisfies the following.

(1) In case that $(\kappa, \bar{\kappa}) \in\left\{(\kappa, 2 n),\left(2 n, 3^{n}-1\right)\right\}$, where the $\kappa$-adjacency is taken from (2.1) with $\kappa \neq 3^{n}-2^{n}-1$ and $\bar{\kappa}$ is the adjacency on $\bar{X}$, then

(a) for each point $x \in X,|X|^{x}$ has exactly one $\kappa$-component $\kappa$-adjacent to $x$;

(b) $|\bar{X}|^{x}$ has exactly two $\bar{\kappa}$-components $\bar{\kappa}$-adjacent to $x$; we denote by $C^{x x}$ and $D^{x x}$ these two components; and

(c) for any point $y \in N_{\kappa}(x) \cap X, N_{\bar{\kappa}}(y) \cap C^{x x} \neq \emptyset$ and $N_{\bar{\kappa}}(y) \cap D^{x x} \neq \emptyset$, where $N_{\kappa}(x)$ means the $\kappa$-neighbors of $x$.

Further, if a closed $\kappa$-surface $X$ does not have a simple $\kappa$-point, then $X$ is called simple.

(2) In case that $(\kappa, \bar{\kappa})=\left(3^{n}-2^{n}-1,2 n\right)$, then

(a) $X$ is $\kappa$-connected,

(b) for each point $x \in X,|X|^{x}$ is a generalized simple closed $\kappa$-curve.

Further, if the image $|X|^{x}$ is a simple closed $\kappa$-curve, then the closed $\kappa$-surface $X$ is called simple.

For a closed $\kappa$-surface $S_{\kappa}$, we denote by $\overline{S_{\kappa}}$ the complement of $S_{\kappa}$ in $\mathbb{Z}^{n}$. Then a point $x$ of $\overline{S_{\kappa}}$ is said to be interior of $S_{\kappa}$ if it belongs to the bounded $\bar{\kappa}$-connected component of $S_{\kappa}$. The set of all interior points of $S_{\kappa}$ is denoted by $\operatorname{int}\left(S_{\kappa}\right)$.

The 3-dimensional digital images $M S S_{18}^{*}$ and $M S S_{6}^{*}$ which are obtained from the minimal simple closed curves $M S C_{8}$ and $M S C_{4}$ in $\mathbb{Z}^{2}$, respectively, are essentially used in establishing the notion of a connected sum [13].

Figure 1. Minimal simple closed curves $M S C_{4}$ and $M S C_{8}$.

- $M S S_{6}^{*}:=M S S_{6} \cup \operatorname{Int}\left(M S S_{6}\right)$ where

$$
M S S_{6} \approx_{(6,6)}\left(M S C_{4} \times[0,2]_{\mathbb{Z}}\right) \cup\left(\operatorname{Int}\left(M S C_{4}\right) \times\{0,2\}\right)
$$

and $\mathrm{MSC}_{4}$ is 4-isomorphic to the set

$$
\{(1,0),(1,1),(0,1),(-1,1),(-1,0),(-1,-1),(0,-1),(1,-1)\} .
$$

- $M S S_{18}^{*}:=M S S_{18} \cup \operatorname{Int}\left(M S S_{18}\right)$ where

$$
M S S_{18} \approx_{(18,18)}\left(M S C_{8} \times\{1\}\right) \cup\left(\operatorname{Int}\left(M S C_{8}\right) \times\{0,2\}\right)
$$

and $M S C_{8}$ is 8 -isomorphic to the set

$$
\{(0,0),(-1,1),(-2,0),(-2,-1),(-1,-2),(0,-1)\} .
$$

2.3. Definition. [13] Let $S_{\kappa_{0}}$ be a closed $\kappa_{0}$-surface in $\mathbb{Z}^{n_{0}}$ and $S_{\kappa_{1}}$ be a closed $\kappa_{1}$-surface in $\mathbb{Z}^{n_{1}}$ for $n_{0}, n_{1} \geq 3$. Consider $A_{\kappa_{0}}^{\prime} \subset A_{\kappa_{0}} \subset S_{\kappa_{0}}$ such that

$$
A_{\kappa_{0}}^{\prime} \approx_{\left(\kappa_{0}, 8\right)} \operatorname{Int}\left(M S C_{8}^{*}\right), A_{\kappa_{0}}^{\prime} \approx_{\left(\kappa_{0}, 4\right)} \operatorname{Int}\left(M S C_{4}^{*}\right) \text { or } A_{\kappa_{0}}^{\prime} \approx_{\left(\kappa_{0}, 8\right)} \operatorname{Int}\left(M S C_{8}^{\prime *}\right) .
$$

Let $f: A_{\kappa_{0}} \rightarrow f\left(A_{\kappa_{0}}\right) \subset S_{\kappa_{1}}$ be a $\left(\kappa_{0}, \kappa_{1}\right)$-isomorphism. Let $S_{\kappa_{i}}^{\prime}=S_{\kappa_{i}} \backslash A_{\kappa_{i}}^{\prime}, i \in\{0,1\}$. Then the connected sum, denoted by $S_{\kappa_{0}} \sharp S_{\kappa_{1}}$, is the quotient space $S_{\kappa_{0}}^{\prime} \cup S_{\kappa_{1}}^{\prime} / \sim$, where $i: A_{\kappa_{0}} \backslash A_{\kappa_{0}}^{\prime} \rightarrow S_{\kappa_{0}}^{\prime}$ is the inclusion map and $i(x) \sim f(x)$ for $x \in A_{\kappa_{0}} \backslash A_{\kappa_{0}}^{\prime}$. 
2.4. Definition. [21] Let $S$ be a set of nonempty subsets of a digital image $(X, \kappa)$. The members of $S$ are called simplexes of $(X, \kappa)$ if the following holds:

( $i$ ) If $p$ and $q$ are distinct points of $s \in S$, then $p$ and $q$ are $\kappa$-adjacent.

(ii) If $s \in S$ and $\emptyset \neq t \subset s$, then $t \in S$ (note this implies every point $p$ that belongs to a simplex determines a simplex $\{p\})$.

An $m$-simplex is a simplex $S$ such that $|S|=m+1$.

Let $P$ be a digital $m$-simplex. If $P^{\prime}$ is a nonempty proper subset of $P$, then $P^{\prime}$ is called a face of $P$.

Since computing homology groups is easier than computing higher degree homotopy groups in algebraic topology, for the same reason computing homology groups of digital images is preferred to computing homotopy groups of digital images. The simplicial homology groups of $n$-dimensional digital images from algebraic topology have been introduced in [1].

2.5. Definition. 1] Let $(X, \kappa)$ be a finite collection of digital $m$-simplices, $0 \leq m \leq d$ for some nonnegative integer $d$. If the following statements hold, then $(X, \kappa)$ is called a finite digital simplicial complex:

(1) If $P$ belongs to $X$, then every face of $P$ also belongs to $X$.

(2) If $P, Q \in X$, then $P \cap Q$ is either empty or a common face of $P$ and $Q$.

The dimension of a digital simplicial complex $X$ is the biggest integer $m$ such that $X$ has an $m$-simplex.

$C_{q}^{\kappa}(X)$ is a free abelian group with basis all digital $(\kappa, q)$-simplices in $X[1]$.

2.6. Corollary. [10] Let $(X, \kappa) \subset \mathbb{Z}^{n}$ be a digital simplicial complex of dimension $m$. Then for all $q>m, C_{q}^{\kappa}(X)$ is a trivial group.

Let $(X, \kappa) \subset \mathbb{Z}^{n}$ be a digital simplicial complex of dimension $m$. The homomorphism $\partial_{q}: C_{q}^{\kappa}(X) \rightarrow C_{q-1}^{\kappa}(X)$ defined by

$$
\partial_{q}\left(<p_{0}, p_{1}, \ldots, p_{q}>\right)= \begin{cases}\sum_{i=0}^{q}(-1)^{i}<p_{0}, p_{1}, \ldots, \widehat{p}_{i}, \ldots, p_{q}>, & q \leq m ; \\ 0, & q>\mathrm{m}\end{cases}
$$

is called a boundary homomorphism where $\widehat{p}_{i}$ means deleting the point $p_{i}$. Then for all $1 \leq q \leq m$, we have $\partial_{q-1} \circ \partial_{q}=0[1]$.

2.7. Theorem. 1$]$ Let $(X, \kappa) \subset \mathbb{Z}^{n}$ be a digital simplicial complex of dimension $m$. Then

$$
C_{*}^{\kappa}(X): 0 \stackrel{\partial_{m+1}}{\longrightarrow} C_{m}^{\kappa}(X) \stackrel{\partial_{m}}{\longrightarrow} C_{m-1}^{\kappa}(X) \stackrel{\partial_{m-1}}{\longrightarrow} \ldots \stackrel{\partial_{1}}{\longrightarrow} C_{0}^{\kappa}(X) \stackrel{\partial_{0}}{\longrightarrow} 0
$$

is a chain complex.

Let $(X, \kappa)$ be a digital simplicial complex. The group of digital simplicial $q$-cycles is $Z_{q}^{\kappa}(X)=\operatorname{Ker} \partial_{q}=\left\{\sigma \in C_{q}^{\kappa}(X) \mid \partial_{q}(\sigma)=0\right\}$ and the group of digital simplicial $q$ boundaries is $B_{q}^{\kappa}(X)=\operatorname{Im} \partial_{q+1}=\left\{\tau \in C_{q}^{\kappa}(X) \mid \partial_{q+1}(\sigma)=\tau\right.$ for $\left.\sigma \in C_{q+1}^{\kappa}(X)\right\}$. The $q$ th digital simplicial homology group is $H_{q}^{\kappa}(X)=Z_{q}^{\kappa}(X) / B_{q}^{\kappa}(X)$ [1].

2.8. Theorem. [1] If $f: X \rightarrow Y$ is a digital $\left(\kappa_{0}, \kappa_{1}\right)$-isomorphism, then for all $q$

$$
H_{q}^{\kappa_{0}}(X) \cong H_{q}^{\kappa_{1}}(Y) \text {. }
$$

2.9. Theorem. [10] Let $(X, \kappa)$ be a directed digital simplicial complex of dimension $m$.

(1) $H_{q}^{\kappa}(X)$ is a finitely generated abelian group for every $q \geq 0$.

(2) $H_{q}^{\kappa}(X)$ is a trivial group for all $q>m$.

(3) $H_{q}^{\kappa}(X)$ is a free abelian group, possibly zero. 
2.10. Definition. 10] Let $(X, \kappa)$ be a digital image of dimension $m$, and for each $q \geq 0$, let $\alpha_{q}$ be the number of digital $(\kappa, q)$-simplexes in $X$. The Euler characteristic of $X$, denoted by $\chi(X, \kappa)$, is defined by

$$
\chi(X, \kappa)=\sum_{q=0}^{m}(-1)^{q} \alpha_{q} .
$$

2.11. Theorem. [10] If $(X, \kappa)$ is a digital image of dimension $m$, then

$$
\chi(X, \kappa)=\sum_{q=0}^{m}(-1)^{q} \operatorname{rank} H_{q}^{\kappa}(X) .
$$

2.12. Example. [10] By the definition of Euler characteristic, we have

$$
\begin{aligned}
& \chi\left(M S S_{6}, 6\right)=\alpha_{0}-\alpha_{1}=26-48=-22 \\
& \chi\left(M S S_{6} \sharp M S S_{6}, 6\right)=\alpha_{0}-\alpha_{1}=42-80=-38 \\
& \chi\left(M S S_{18}, 18\right)=\alpha_{0}-\alpha_{1}+\alpha_{2}=10-20+8=-2 \\
& \chi\left(M S S_{18} \sharp M S S_{18}, 18\right)=\alpha_{0}-\alpha_{1}+\alpha_{2}=14-28+8=-6
\end{aligned}
$$

\section{MAIN RESULTS}

Simplicial homology groups of several digital surfaces have been computed in [10]. By using an argument similar to that of [10, we have the following theorems.

3.1. Theorem. The digital simplicial homology groups of $M S S_{18} \sharp M S S_{18}$ are

$$
H_{q}^{18}\left(M S S_{18} \sharp M S S_{18}\right)= \begin{cases}\mathbb{Z}, & q=0 ; \\ \mathbb{Z}^{7}, & q=1 ; \\ 0, & q \geq 2 .\end{cases}
$$

Figure 2. $M S S_{18} \sharp M S S_{18}$

Proof. Let

$$
\begin{aligned}
M S S_{18} \sharp M S S_{18}=\left\{c_{0}\right. & =(1,0,1), c_{1}=(1,1,1), c_{2}=(1,2,1), \\
c_{3} & =(0,3,1), c_{4}=(-1,2,1), c_{5}=(-1,1,1), \\
c_{6} & =(-1,0,1), c_{7}=(0,-1,1), c_{8}=(0,2,2), \\
c_{9} & =(0,1,2), c_{10}=(0,0,2), c_{11}=(0,2,0), \\
& \left.c_{12}=(0,1,0), c_{13}=(0,0,0)\right\} .
\end{aligned}
$$

Then we can direct $M S S_{18} \sharp M S S_{18}$ by the ordering $c_{6}<c_{5}<c_{4}<c_{7}<c_{13}<c_{10}<$ $c_{12}<c_{9}<c_{11}<c_{8}<c_{3}<c_{0}<c_{1}<c_{2}$. We have the following simplicial chain complexes:

$C_{0}^{18}\left(M S S_{18} \sharp M S S_{18}\right)$ has for a basis $\left\{\left\langle c_{0}\right\rangle,\left\langle c_{1}\right\rangle, \ldots,\left\langle c_{13}\right\rangle\right\}$, $C_{1}^{18}\left(M S S_{18} \sharp M S S_{18}\right)$ has for a basis

$$
\begin{aligned}
& \left\{\left\langle c_{7} c_{0}\right\rangle,\left\langle c_{10} c_{0}\right\rangle,\left\langle c_{13} c_{0}\right\rangle,\left\langle c_{0} c_{1}\right\rangle,\left\langle c_{9} c_{1}\right\rangle,\left\langle c_{12} c_{1}\right\rangle,\left\langle c_{1} c_{2}\right\rangle,\left\langle c_{8} c_{2}\right\rangle,\left\langle c_{11} c_{2}\right\rangle,\left\langle c_{3} c_{2}\right\rangle,\left\langle c_{4} c_{3}\right\rangle,\right. \\
& \quad\left\langle c_{8} c_{3}\right\rangle,\left\langle c_{11} c_{3}\right\rangle,\left\langle c_{5} c_{4}\right\rangle,\left\langle c_{4} c_{8}\right\rangle,\left\langle c_{4} c_{11}\right\rangle,\left\langle c_{6} c_{5}\right\rangle,\left\langle c_{5} c_{9}\right\rangle,\left\langle c_{5} c_{12}\right\rangle,\left\langle c_{6} c_{7}\right\rangle,\left\langle c_{6} c_{10}\right\rangle,\left\langle c_{6} c_{13}\right\rangle, \\
& \left.\quad\left\langle c_{7} c_{10}\right\rangle,\left\langle c_{7} c_{13}\right\rangle,\left\langle c_{9} c_{8}\right\rangle,\left\langle c_{10} c_{9}\right\rangle,\left\langle c_{12} c_{11}\right\rangle,\left\langle c_{13} c_{12}\right\rangle\right\},
\end{aligned}
$$

and $C_{2}^{18}\left(M S S_{18} \sharp M S S_{18}\right)$ has for a basis

$$
\left\{\left\langle c_{7} c_{13} c_{0}\right\rangle,\left\langle c_{7} c_{10} c_{0}\right\rangle,\left\langle c_{8} c_{3} c_{2}\right\rangle,\left\langle c_{11} c_{3} c_{2}\right\rangle,\left\langle c_{4} c_{8} c_{3}\right\rangle,\left\langle c_{4} c_{11} c_{3}\right\rangle,\left\langle c_{6} c_{7} c_{10}\right\rangle,\left\langle c_{6} c_{7} c_{13}\right\rangle\right\} .
$$


Thus, we obtain the following short sequence:

$$
0 \stackrel{\partial_{3}}{\longrightarrow} C_{2}^{18}\left(M S S_{18} \sharp M S S_{18}\right) \stackrel{\partial_{2}}{\longrightarrow} C_{1}^{18}\left(M S S_{18} \sharp M S S_{18}\right) \stackrel{\partial_{1}}{\longrightarrow} C_{0}^{18}\left(M S S_{18} \sharp M S S_{18}\right) \stackrel{\partial_{0}}{\longrightarrow} 0 .
$$

By Theorem 2.9, $H_{q}^{18}\left(M S S_{18} \sharp M S S_{18}\right)$ is a trivial group for all $q>2$.

We determine the kernel of $\partial_{2}$. If

$$
\begin{aligned}
\partial_{2} & a_{1}\left\langle c_{7} c_{13} c_{0}\right\rangle+a_{2}\left\langle c_{7} c_{10} c_{0}\right\rangle+a_{3}\left\langle c_{8} c_{3} c_{2}\right\rangle+a_{4}\left\langle c_{11} c_{3} c_{2}\right\rangle+a_{5}\left\langle c_{4} c_{8} c_{3}\right\rangle+a_{6}\left\langle c_{4} c_{11} c_{3}\right\rangle \\
& \left.+a_{7}\left\langle c_{6} c_{7} c_{10}\right\rangle+a_{8}\left\langle c_{6} c_{7} c_{13}\right\rangle\right)=a_{1}\left\langle c_{13} c_{0}\right\rangle+\left(-a_{1}-a_{2}\right)\left\langle c_{7} c_{0}\right\rangle+\left(a_{1}+a_{8}\right)\left\langle c_{7} c_{13}\right\rangle \\
& +a_{2}\left\langle c_{10} c_{0}\right\rangle+\left(a_{2}+a_{7}\right)\left\langle c_{7} c_{10}\right\rangle+\left(a_{3}+a_{4}\right)\left\langle c_{3} c_{2}\right\rangle-a_{3}\left\langle c_{8} c_{2}\right\rangle+\left(a_{3}+a_{5}\right)\left\langle c_{8} c_{3}\right\rangle \\
& -a_{4}\left\langle c_{11} c_{2}\right\rangle+\left(a_{4}+a_{6}\right)\left\langle c_{11} c_{3}\right\rangle+\left(-a_{5}-a_{6}\right)\left\langle c_{4} c_{3}\right\rangle+a_{5}\left\langle c_{4} c_{8}\right\rangle \\
& +a_{6}\left\langle c_{4} c_{11}\right\rangle-a_{7}\left\langle c_{6} c_{10}\right\rangle+\left(a_{7}+a_{8}\right)\left\langle c_{6} c_{7}\right\rangle-a_{8}\left\langle c_{6} c_{13}\right\rangle=0,
\end{aligned}
$$

then one easily sees that $a_{1}=a_{2}=a_{3}=a_{4}=a_{5}=a_{6}=a_{7}=a_{8}=0$. Therefore, $Z_{2}^{18}\left(M S S_{18} \sharp M S S_{18}\right)=\{0\}$ and hence $H_{2}^{18}\left(M S S_{18} \sharp M S S_{18}\right)=\{0\}$.

Since $\operatorname{Ker} \partial_{2}=Z_{2}^{18}\left(M S S_{18} \sharp M S S_{18}\right)=\{0\}, \operatorname{Im} \partial_{2} \cong C_{2}^{8}\left(M S S_{18} \sharp M S S_{18}\right)$, and so $B_{1}^{18}\left(M S S_{18} \sharp M S S_{18}\right) \cong \mathbb{Z}^{8}$.

We can use standard methods to determine that $Z_{1}^{18}\left(M S S_{18} \sharp M S S_{18}\right) \cong \mathbb{Z}^{15}$, from which it follows easily that $B_{0}^{18}\left(M S S_{18} \sharp M S S_{18}\right) \cong \mathbb{Z}^{13}$. However, the direct calculation of $Z_{1}^{18}\left(M S S_{18} \sharp M S S_{18}\right)$ is very long. Since our goal is to calculate $H_{1}^{18}\left(M S S_{18} \sharp M S S_{18}\right)$, we will do so below without showing a direct calculation of $Z_{1}^{18}\left(M S S_{18} \sharp M S S_{18}\right)$.

By using the short sequence again, we have

$$
Z_{0}^{18}\left(M S S_{18} \sharp M S S_{18}\right)=\left\{\sum_{i=0}^{13} a_{i}\left\langle c_{i}\right\rangle \mid a_{i} \in \mathbb{Z}, i=0,1, \ldots, 13\right\} \cong \mathbb{Z}^{14}
$$

Any 0-cycle $w_{0}=\sum_{i=0}^{13} a_{i}\left\langle c_{i}\right\rangle$ can be written as

$$
\begin{aligned}
& w_{0}=\partial_{1}(\left(-a_{7}\right)\left\langle c_{7} c_{0}\right\rangle+\left(a_{1}+a_{2}+a_{3}\right)\left\langle c_{0} c_{1}\right\rangle+\left(a_{2}+a_{3}\right)\left\langle c_{1} c_{2}\right\rangle \\
&+\left(-a_{3}\right)\left\langle c_{3} c_{2}\right\rangle+a_{11}\left\langle c_{4} c_{11}\right\rangle+\left(a_{4}+a_{11}\right)\left\langle c_{5} c_{4}\right\rangle+a_{12}\left\langle c_{5} c_{12}\right\rangle \\
&+\left(a_{4}+a_{5}+a_{11}+a_{12}\right)\left\langle c_{6} c_{5}\right\rangle+a_{13}\left\langle c_{6} c_{13}\right\rangle \\
&+\left(-a_{4}-a_{5}-a_{6}-a_{11}-a_{12}-a_{13}\right)\left\langle c_{6} c_{10}\right\rangle+a_{8}\left\langle c_{9} c_{8}\right\rangle \\
&\left.+\left(a_{8}+a_{9}\right)\left\langle c_{10} c_{9}\right\rangle+\left(a_{0}+a_{1}+a_{2}+a_{3}+a_{7}\right)\left\langle c_{10} c_{0}\right\rangle\right)+\sum_{i=0}^{13} a_{i}\left\langle c_{10}\right\rangle .
\end{aligned}
$$

So $w_{0}$ is homologous to 0 -chain $\sum_{i=0}^{13} a_{i}\left\langle c_{10}\right\rangle$. Hence the 0-chain is homologous to an integral multiple of $\left\langle c_{10}\right\rangle$. Thus we deduce $H_{0}^{18}\left(M S S_{18} \sharp M S S_{18}\right) \cong \mathbb{Z}$.

To compute the $H_{1}^{18}\left(M S S_{18} \sharp M S S_{18}\right)$, we can use the results in [10]. By Example 2.12 , we know that $\chi\left(M S S_{18} \sharp M S S_{18}, 18\right)=-6$. From Theorem 2.11,

$$
\begin{aligned}
\chi\left(M S S_{18} \sharp M S S_{18}, 18\right) & =\sum_{q=0}^{2}(-1)^{q} \operatorname{rank} H_{q}^{18}\left(M S S_{18} \sharp M S S_{18}\right) \\
-6 & =1-\operatorname{rank} H_{1}^{18}\left(M S S_{18} \sharp M S S_{18}\right)+0
\end{aligned}
$$

Thus we get $\operatorname{rank} H_{1}^{18}\left(M S S_{18} \sharp M S S_{18}\right)=7$ which in turn gives us

$$
H_{1}^{18}\left(M S S_{18} \sharp M S S_{18}\right) \cong \mathbb{Z}^{7} \text {. }
$$

3.2. Theorem. The digital simplicial homology groups of $M S S_{6}$ are 


$$
H_{q}^{6}\left(M S S_{6}\right)= \begin{cases}\mathbb{Z}, & q=0 \\ \mathbb{Z}^{23}, & q=1 \\ 0, & q \neq 0,1\end{cases}
$$

Figure 3. $M S S_{6}$

Proof. If we take

$$
\begin{aligned}
M S S_{6}=\left\{c_{0}\right. & =(-1,-1,0), c_{1}=(0,-1,0), c_{2}=(1,-1,0), c_{3}=(1,0,0), \\
& c_{4}=(0,0,0), c_{5}=(-1,0,0), c_{6}=(-1,1,0), c_{7}=(0,1,0), \\
& c_{8}=(1,1,0), c_{9}=(1,1,1), c_{10}=(0,1,1), c_{11}=(-1,1,1), \\
& c_{12}=(-1,0,1), c_{13}=(1,0,1), c_{14}=(1,-1,1), c_{15}=(0,-1,1), \\
& c_{16}=(-1,-1,1), c_{17}=(-1,-1,2), c_{18}=(0,-1,2), c_{19}=(1,-1,2), \\
& c_{20}=(1,0,2), c_{21}=(0,0,2), c_{22}=(-1,0,2), c_{23}=(-1,1,2), \\
& \left.c_{24}=(0,1,2), c_{25}=(1,1,2)\right\},
\end{aligned}
$$

then we can direct $M S S_{6}$ by the ordering $c_{0}<c_{16}<c_{17}<c_{5}<c_{12}<c_{22}<c_{6}<c_{11}<$ $c_{23}<c_{1}<c_{15}<c_{18}<c_{4}<c_{21}<c_{7}<c_{10}<c_{24}<c_{2}<c_{14}<c_{19}<c_{3}<c_{13}<c_{20}<$ $c_{8}<c_{9}<c_{25}$.

We have the following simplicial chain complexes: $C_{0}^{6}\left(M S S_{6}\right)$ has for a basis $\left\{\left\langle c_{0}\right\rangle,\left\langle c_{1}\right\rangle, \ldots,\left\langle c_{25}\right\rangle\right\}$, and $C_{1}^{6}\left(M S S_{6}\right)$ has for a basis

$$
\begin{aligned}
& \left\{\left\langle c_{0} c_{1}\right\rangle,\left\langle c_{0} c_{5}\right\rangle,\left\langle c_{0} c_{16}\right\rangle,\left\langle c_{1} c_{2}\right\rangle,\left\langle c_{1} c_{4}\right\rangle,\left\langle c_{1} c_{15}\right\rangle,\left\langle c_{2} c_{14}\right\rangle,\left\langle c_{2} c_{3}\right\rangle,\left\langle c_{4} c_{3}\right\rangle,\left\langle c_{3} c_{8}\right\rangle,\left\langle c_{3} c_{13}\right\rangle,\right. \\
& \left\langle c_{5} c_{4}\right\rangle,\left\langle c_{4} c_{7}\right\rangle,\left\langle c_{5} c_{6}\right\rangle,\left\langle c_{5} c_{12}\right\rangle,\left\langle c_{6} c_{7}\right\rangle,\left\langle c_{6} c_{11}\right\rangle,\left\langle c_{7} c_{8}\right\rangle,\left\langle c_{7} c_{10}\right\rangle,\left\langle c_{8} c_{9}\right\rangle,\left\langle c_{10} c_{9}\right\rangle,\left\langle c_{13} c_{9}\right\rangle, \\
& \left\langle c_{9} c_{25}\right\rangle,\left\langle c_{11} c_{10}\right\rangle,\left\langle c_{10} c_{24}\right\rangle,\left\langle c_{12} c_{11}\right\rangle,\left\langle c_{11} c_{23}\right\rangle,\left\langle c_{16} c_{12}\right\rangle,\left\langle c_{12} c_{22}\right\rangle,\left\langle c_{14} c_{13}\right\rangle,\left\langle c_{13} c_{20}\right\rangle, \\
& \left\langle c_{15} c_{14}\right\rangle,\left\langle c_{14} c_{19}\right\rangle,\left\langle c_{16} c_{15}\right\rangle,\left\langle c_{15} c_{18}\right\rangle,\left\langle c_{16} c_{17}\right\rangle,\left\langle c_{17} c_{18}\right\rangle,\left\langle c_{17} c_{22}\right\rangle,\left\langle c_{18} c_{19}\right\rangle,\left\langle c_{18} c_{21}\right\rangle, \\
& \left.\left\langle c_{19} c_{20}\right\rangle,\left\langle c_{21} c_{20}\right\rangle,\left\langle c_{20} c_{25}\right\rangle,\left\langle c_{22} c_{21}\right\rangle,\left\langle c_{21} c_{24}\right\rangle,\left\langle c_{22} c_{23}\right\rangle,\left\langle c_{23} c_{24}\right\rangle,\left\langle c_{24} c_{25}\right\rangle\right\} .
\end{aligned}
$$

Thus we get the following short sequence:

$$
0 \stackrel{\partial_{2}}{\longrightarrow} C_{1}^{6}\left(M S S_{6}\right) \stackrel{\partial_{1}}{\longrightarrow} C_{0}^{6}\left(M S S_{6}\right) \stackrel{\partial_{0}}{\longrightarrow} 0 .
$$

By Theorem 2.9, we have $H_{q}^{6}\left(M S S_{6}\right)=\{0\}$ for every $q>1$.

Direct calculation yields that $Z_{1}^{6}\left(M S S_{6}\right) \cong \mathbb{Z}^{23}$, from which it follows easily that $B_{0}^{6}\left(M S S_{6}\right) \cong \mathbb{Z}^{25}$. However, direct calculation of $Z_{1}^{6}\left(M S S_{6}\right)$ is very long. Since our goal is to calculate $H_{1}^{6}\left(M S S_{6}\right)$, we do so below without showing a direct calculation of $Z_{1}^{6}\left(M S S_{6}\right)$.

By using the short sequence, we have

$$
Z_{0}^{6}\left(M S S_{6}\right)=\left\{\sum_{i=0}^{25} a_{i}\left\langle c_{i}\right\rangle \mid a_{i} \in \mathbb{Z}, i=0,1, \ldots, 25\right\} \cong \mathbb{Z}^{26}
$$

Any 0-cycle $w_{0}=\sum_{i=0}^{25} a_{i}\left\langle c_{i}\right\rangle$ can be written as 


$$
\begin{aligned}
w_{0}=\partial_{1} & \left(-a_{6}\right)\left\langle c_{6} c_{11}\right\rangle+\left(-a_{6}-a_{11}\right)\left\langle c_{11} c_{23}\right\rangle+\left(a_{6}+a_{11}+a_{23}\right)\left\langle c_{22} c_{23}\right\rangle \\
& +\left(a_{6}+a_{11}+a_{22}+a_{23}\right)\left\langle c_{12} c_{22}\right\rangle+\left(a_{6}+a_{11}+a_{12}+a_{22}+a_{23}\right)\left\langle c_{5} c_{12}\right\rangle \\
& +\left(a_{5}+a_{6}+a_{11}+a_{12}+a_{22}+a_{23}\right)\left\langle c_{0} c_{5}\right\rangle \\
& +\left(-a_{0}-a_{5}-a_{6}-a_{11}-a_{12}-a_{22}-a_{23}\right)\left\langle c_{0} c_{16}\right\rangle \\
& +\left(-a_{0}-a_{5}-a_{6}-a_{11}-a_{12}-a_{16}-a_{22}-a_{23}\right)\left\langle c_{16} c_{17}\right\rangle \\
& +\left(-a_{0}-a_{5}-a_{6}-a_{11}-a_{12}-a_{16}-a_{17}-a_{22}-a_{23}\right)\left\langle c_{17} c_{18}\right\rangle \\
& +a_{15}\left\langle c_{1} c_{15}\right\rangle+\left(-a_{1}-a_{15}\right)\left\langle c_{1} c_{4}\right\rangle \\
& +\left(-a_{1}-a_{4}-a_{15}\right)\left\langle c_{4} c_{7}\right\rangle+\left(-a_{1}-a_{4}-a_{7}-a_{15}\right)\left\langle c_{7} c_{10}\right\rangle \\
& +\left(-a_{1}-a_{4}-a_{7}-a_{10}-a_{15}\right)\left\langle c_{10} c_{24}\right\rangle \\
& +\left(a_{1}+a_{4}+a_{7}+a_{10}+a_{15}+a_{24}\right)\left\langle c_{21} c_{24}\right\rangle \\
& +\left(a_{1}+a_{4}+a_{7}+a_{10}+a_{15}+a_{21}+a_{24}\right)\left\langle c_{18} c_{21}\right\rangle+\left(-a_{8}\right)\left\langle c_{8} c_{9}\right\rangle \\
& +\left(-a_{8}-a_{9}\right)\left\langle c_{9} c_{25}\right\rangle+\left(a_{8}+a_{9}+a_{25}\right)\left\langle c_{20} c_{25}\right\rangle \\
& +\left(a_{8}+a_{9}+a_{20}+a_{25}\right)\left\langle c_{13} c_{20}\right\rangle+\left(a_{8}+a_{9}+a_{13}+a_{20}+a_{25}\right)\left\langle c_{3} c_{13}\right\rangle \\
& +\left(a_{3}+a_{8}+a_{9}+a_{13}+a_{20}+a_{25}\right)\left\langle c_{2} c_{3}\right\rangle \\
& +\left(-a_{2}-a_{3}-a_{8}-a_{9}-a_{13}-a_{20}-a_{25}\right)\left\langle c_{2} c_{14}\right\rangle \\
& +\left(-a_{2}-a_{3}-a_{8}-a_{9}-a_{13}-a_{14}-a_{20}-a_{25}\right)\left\langle c_{14} c_{19}\right\rangle \\
& \left.+\left(a_{2}+a_{3}+a_{8}+a_{9}+a_{13}+a_{14}+a_{19}+a_{20}+a_{25}\right)\left\langle c_{18} c_{19}\right\rangle\right)+\sum_{i=0}^{25} a_{i}\left\langle c_{18}\right\rangle .
\end{aligned}
$$

So $w_{0}$ is homologous to 0 -chain $\sum_{i=0}^{25} a_{i}\left\langle c_{18}\right\rangle$. Hence the 0 -chain is homologous to an integral multiple of $\left\langle c_{18}\right\rangle$. Thus we get

$$
H_{0}^{6}\left(M S S_{6}\right) \cong \mathbb{Z}
$$

We use the results in [10] to compute the $H_{1}^{6}\left(M S S_{6}\right)$. From Example 2.12, we have $\chi\left(M S S_{6}, 6\right)=-22$. From Theorem 2.11,

$$
\begin{aligned}
\chi\left(M S S_{6}, 6\right) & =\sum_{q=0}^{1}(-1)^{q} \operatorname{rank} H_{q}^{6}\left(M S S_{6}\right) \\
-22 & =1-\operatorname{rank} H_{1}^{6}\left(M S S_{6}\right)
\end{aligned}
$$

Thus we get $\operatorname{rank} H_{1}^{6}\left(M S S_{6}\right)=23$ which gives us

$$
H_{1}^{6}\left(M S S_{6}\right) \cong \mathbb{Z}^{23} \text {. }
$$

3.3. Theorem. The digital simplicial homology groups of $M S S_{6} \sharp M S S_{6}$ are

$$
H_{q}^{6}\left(M S S_{6} \sharp M S S_{6}\right)= \begin{cases}\mathbb{Z}, & q=0 ; \\ \mathbb{Z}^{39}, & q=1 ; \\ 0, & q \neq 0,1 .\end{cases}
$$


Figure 4. $M S S_{6} \sharp M S S_{6}$

Proof. Let

$$
\begin{aligned}
M S S_{6} \sharp M S S_{6}=\left\{c_{0}\right. & =(0,0,0), c_{1}=(1,0,0), c_{2}=(2,0,0), c_{3}=(2,1,0), \\
& c_{4}=(1,1,0), c_{5}=(0,1,0), c_{6}=(0,2,0), c_{7}=(1,2,0), \\
& c_{8}=(2,2,0), c_{9}=(2,3,0), c_{10}=(1,3,0), c_{11}=(0,3,0), \\
& c_{12}=(0,4,0), c_{13}=(1,4,0), c_{14}=(2,4,0), c_{15}=(2,4,1), \\
& c_{16}=(1,4,1), c_{17}=(0,4,1), c_{18}=(0,3,1), c_{19}=(2,3,1), \\
& c_{20}=(2,2,1), c_{21}=(0,2,1), c_{22}=(0,1,1), c_{23}=(2,1,1), \\
& c_{24}=(2,0,1), c_{25}=(1,0,1), c_{26}=(0,0,1), c_{27}=(0,0,2), \\
& c_{28}=(1,0,2), c_{29}=(2,0,2), c_{30}=(2,1,2), c_{31}=(1,1,2), \\
& c_{32}=(0,1,2), c_{33}=(0,2,2), c_{34}=(1,2,2), c_{35}=(2,2,2), \\
& c_{36}=(2,3,2), c_{37}=(1,3,2), c_{38}=(0,3,2), c_{39}=(0,4,2), \\
c_{40} & \left.=(1,4,2), c_{41}=(2,4,2)\right\} .
\end{aligned}
$$

We can direct $M S S_{6} \sharp M S S_{6}$ by the ordering $c_{0}<c_{26}<c_{27}<c_{5}<c_{22}<c_{32}<c_{6}<$ $c_{21}<c_{33}<c_{11}<c_{18}<c_{38}<c_{12}<c_{17}<c_{39}<c_{1}<c_{25}<c_{28}<c_{4}<c_{31}<c_{7}<c_{34}<$ $c_{10}<c_{37}<c_{13}<c_{16}<c_{40}<c_{2}<c_{24}<c_{29}<c_{3}<c_{23}<c_{30}<c_{8}<c_{20}<c_{35}<c_{9}<$ $c_{19}<c_{36}<c_{14}<c_{15}<c_{41}$.

We have the following simplicial chain complexes:

$C_{0}^{6}\left(M S S_{6} \sharp M S S_{6}\right)$ has for a basis $\left\{\left\langle c_{0}\right\rangle,\left\langle c_{1}\right\rangle, \ldots,\left\langle c_{41}\right\rangle\right\}$, and $C_{1}^{6}\left(M S S_{6} \sharp M S S_{6}\right)$ has for a basis

$\left\{\left\langle c_{0} c_{1}\right\rangle,\left\langle c_{0} c_{5}\right\rangle,\left\langle c_{0} c_{26}\right\rangle,\left\langle c_{1} c_{4}\right\rangle,\left\langle c_{1} c_{2}\right\rangle,\left\langle c_{1} c_{25}\right\rangle,\left\langle c_{2} c_{3}\right\rangle,\left\langle c_{2} c_{24}\right\rangle,\left\langle c_{4} c_{3}\right\rangle,\left\langle c_{3} c_{8}\right\rangle,\left\langle c_{3} c_{23}\right\rangle\right.$, $\left\langle c_{4} c_{7}\right\rangle,\left\langle c_{5} c_{4}\right\rangle,\left\langle c_{5} c_{6}\right\rangle,\left\langle c_{5} c_{22}\right\rangle,\left\langle c_{6} c_{11}\right\rangle,\left\langle c_{6} c_{21}\right\rangle,\left\langle c_{6} c_{7}\right\rangle,\left\langle c_{7} c_{10}\right\rangle,\left\langle c_{7} c_{8}\right\rangle,\left\langle c_{8} c_{9}\right\rangle,\left\langle c_{8} c_{20}\right\rangle$, $\left\langle c_{9} c_{14}\right\rangle,\left\langle c_{10} c_{9}\right\rangle,\left\langle c_{9} c_{19}\right\rangle,\left\langle c_{10} c_{13}\right\rangle,\left\langle c_{11} c_{10}\right\rangle,\left\langle c_{11} c_{12}\right\rangle,\left\langle c_{11} c_{18}\right\rangle,\left\langle c_{12} c_{13}\right\rangle,\left\langle c_{12} c_{17}\right\rangle$, $\left\langle c_{13} c_{16}\right\rangle,\left\langle c_{13} c_{14}\right\rangle,\left\langle c_{14} c_{15}\right\rangle,\left\langle c_{16} c_{15}\right\rangle,\left\langle c_{19} c_{15}\right\rangle,\left\langle c_{15} c_{41}\right\rangle,\left\langle c_{17} c_{16}\right\rangle,\left\langle c_{16} c_{40}\right\rangle,\left\langle c_{18} c_{17}\right\rangle$, $\left\langle c_{17} c_{39}\right\rangle,\left\langle c_{21} c_{18}\right\rangle,\left\langle c_{18} c_{38}\right\rangle,\left\langle c_{20} c_{19}\right\rangle,\left\langle c_{19} c_{36}\right\rangle,\left\langle c_{23} c_{20}\right\rangle,\left\langle c_{20} c_{35}\right\rangle,\left\langle c_{22} c_{21}\right\rangle,\left\langle c_{21} c_{33}\right\rangle$, $\left\langle c_{26} c_{22}\right\rangle,\left\langle c_{22} c_{32}\right\rangle,\left\langle c_{24} c_{23}\right\rangle,\left\langle c_{23} c_{30}\right\rangle,\left\langle c_{25} c_{24}\right\rangle,\left\langle c_{24} c_{29}\right\rangle,\left\langle c_{26} c_{25}\right\rangle,\left\langle c_{25} c_{28}\right\rangle,\left\langle c_{26} c_{27}\right\rangle$, $\left\langle c_{27} c_{28}\right\rangle,\left\langle c_{27} c_{32}\right\rangle,\left\langle c_{28} c_{29}\right\rangle,\left\langle c_{28} c_{31}\right\rangle,\left\langle c_{29} c_{30}\right\rangle,\left\langle c_{30} c_{35}\right\rangle,\left\langle c_{31} c_{30}\right\rangle,\left\langle c_{32} c_{31}\right\rangle,\left\langle c_{31} c_{34}\right\rangle$, $\left\langle c_{32} c_{33}\right\rangle,\left\langle c_{33} c_{34}\right\rangle,\left\langle c_{33} c_{38}\right\rangle,\left\langle c_{34} c_{35}\right\rangle,\left\langle c_{34} c_{37}\right\rangle,\left\langle c_{35} c_{36}\right\rangle,\left\langle c_{31} c_{34}\right\rangle,\left\langle c_{32} c_{33}\right\rangle,\left\langle c_{33} c_{34}\right\rangle$, $\left\langle c_{33} c_{38}\right\rangle,\left\langle c_{34} c_{35}\right\rangle,\left\langle c_{34} c_{37}\right\rangle,\left\langle c_{35} c_{36}\right\rangle,\left\langle c_{31} c_{34}\right\rangle,\left\langle c_{32} c_{33}\right\rangle,\left\langle c_{33} c_{34}\right\rangle,\left\langle c_{33} c_{38}\right\rangle,\left\langle c_{34} c_{35}\right\rangle$, $\left\langle c_{34} c_{37}\right\rangle,\left\langle c_{35} c_{36}\right\rangle,\left\langle c_{27} c_{32}\right\rangle,\left\langle c_{28} c_{29}\right\rangle,\left\langle c_{28} c_{31}\right\rangle,\left\langle c_{29} c_{30}\right\rangle,\left\langle c_{30} c_{35}\right\rangle,\left\langle c_{31} c_{30}\right\rangle,\left\langle c_{32} c_{31}\right\rangle$, $\left\langle c_{31} c_{34}\right\rangle,\left\langle c_{32} c_{33}\right\rangle,\left\langle c_{33} c_{34}\right\rangle,\left\langle c_{33} c_{38}\right\rangle,\left\langle c_{34} c_{35}\right\rangle,\left\langle c_{34} c_{37}\right\rangle,\left\langle c_{35} c_{36}\right\rangle,\left\langle c_{37} c_{36}\right\rangle,\left\langle c_{36} c_{41}\right\rangle$, $\left.\left\langle c_{38} c_{37}\right\rangle,\left\langle c_{37} c_{40}\right\rangle,\left\langle c_{38} c_{39}\right\rangle,\left\langle c_{39} c_{40}\right\rangle,\left\langle c_{40} c_{41}\right\rangle\right\}$.

Thus we obtain the following short sequence:

$$
0 \stackrel{\partial_{2}}{\longrightarrow} C_{1}^{6}\left(M S S_{6} \sharp M S S_{6}\right) \stackrel{\partial_{1}}{\longrightarrow} C_{0}^{6}\left(M S S_{6} \sharp M S S_{6}\right) \stackrel{\partial_{0}}{\longrightarrow} 0 .
$$

By Theorem 2.9, $H_{q}^{6}\left(M S S_{6} \sharp M S S_{6}\right)$ is a trivial group for $q>1$.

Direct calculation yields that $Z_{1}^{6}\left(M S S_{6} \sharp M S S_{6}\right) \cong \mathbb{Z}^{39}$, from which it follows easily that $B_{0}^{6}\left(M S S_{6} \sharp M S S_{6}\right) \cong \mathbb{Z}^{41}$. However, direct calculation of the group $Z_{1}^{6}\left(M S S_{6} \sharp M S S_{6}\right)$ 
of digital simplicial 1-cylces is very long. Since our goal is to calculate $H_{1}^{6}\left(M S S_{6} \sharp M S S_{6}\right)$, we do so below without showing a direct calculation of $Z_{1}^{6}\left(M S S_{6} \sharp M S S_{6}\right)$.

By using the short sequence again, we have

$$
Z_{0}^{6}\left(M S S_{6} \sharp M S S_{6}\right)=\left\{\sum_{i=0}^{41} a_{i}\left\langle c_{i}\right\rangle \mid a_{i} \in \mathbb{Z}, i=0,1, \ldots, 41\right\} \cong \mathbb{Z}^{42} .
$$

Any 0 -cycle $w_{0}=\sum_{i=0}^{41} a_{i}\left\langle c_{i}\right\rangle$ can be written as

$$
\begin{aligned}
w_{0}=\partial_{1}( & -a_{12}\left\langle c_{12} c_{17}\right\rangle+\left(-a_{12}-a_{17}\right)\left\langle c_{17} c_{39}\right\rangle+\left(a_{12}+a_{17}+a_{39}\right)\left\langle c_{38} c_{39}\right\rangle \\
& +\left(a_{12}+a_{17}+a_{38}+a_{39}\right)\left\langle c_{18} c_{38}\right\rangle \\
& +\left(a_{12}+a_{17}+a_{18}+a_{38}+a_{39}\right)\left\langle c_{11} c_{18}\right\rangle \\
& +\left(a_{11}+a_{12}+a_{17}+a_{18}+a_{38}+a_{39}\right)\left\langle c_{6} c_{11}\right\rangle \\
& +\left(-a_{6}-a_{11}-a_{12}-a_{17}-a_{18}-a_{38}-a_{39}\right)\left\langle c_{6} c_{21}\right\rangle \\
& +\left(-a_{6}-a_{11}-a_{12}-a_{17}-a_{18}-a_{21}-a_{38}-a_{39}\right)\left\langle c_{21} c_{33}\right\rangle \\
& +\left(a_{6}+a_{11}+a_{12}+a_{17}+a_{18}+a_{21}+a_{33}+a_{38}+a_{39}\right)\left\langle c_{32} c_{33}\right\rangle \\
& +\left(a_{6}+a_{11}+a_{12}+a_{17}+a_{18}+a_{21}+a_{32}+a_{33}+a_{38}+a_{39}\right)\left\langle c_{22} c_{32}\right\rangle
\end{aligned}
$$




$$
\begin{aligned}
& +\left(a_{6}+a_{11}+a_{12}+a_{17}+a_{18}+a_{21}+a_{22}+a_{32}+a_{33}+a_{38}+a_{39}\right)\left\langle c_{5} c_{22}\right\rangle \\
& +\left(a_{5}+a_{6}+a_{11}+a_{12}+a_{17}+a_{18}+a_{21}+a_{22}+a_{32}+a_{33}\right. \\
& \left.+a_{38}+a_{39}\right)\left\langle c_{0} c_{5}\right\rangle+\left(-a_{0}-a_{5}-a_{6}-a_{11}-a_{12}-a_{17}-a_{18}-a_{21}\right. \\
& \left.-a_{22}-a_{32}-a_{33}-a_{38}-a_{39}\right)\left\langle c_{0} c_{26}\right\rangle+\left(-a_{0}-a_{5}-a_{6}-a_{11}-\right. \\
& \left.-a_{12}-a_{17}-a_{18}-a_{21}-a_{22}-a_{26}-a_{32}-a_{33}-a_{38}-a_{39}\right)\left\langle c_{26} c_{27}\right\rangle \\
& +\left(-a_{0}-a_{5}-a_{6}-a_{11}-a_{12}-a_{17}-a_{18}-a_{21}-a_{22}-a_{26}-a_{27}-a_{32}\right. \\
& \left.-a_{33}-a_{38}-a_{39}\right)\left\langle c_{27} c_{28}\right\rangle+a_{25}\left\langle c_{1} c_{25}\right\rangle+\left(-a_{1}-a_{25}\right)\left\langle c_{1} c_{4}\right\rangle \\
& +\left(-a_{1}-a_{4}-a_{25}\right)\left\langle c_{4} c_{7}\right\rangle+\left(-a_{1}-a_{4}-a_{7}-a_{25}\right)\left\langle c_{7} c_{10}\right\rangle \\
& +\left(-a_{1}-a_{4}-a_{7}-a_{10}-a_{25}\right)\left\langle c_{10} c_{13}\right\rangle \\
& +\left(-a_{1}-a_{4}-a_{7}-a_{10}-a_{13}-a_{25}\right)\left\langle c_{13} c_{16}\right\rangle \\
& +\left(-a_{1}-a_{4}-a_{7}-a_{10}-a_{13}-a_{16}-a_{25}\right)\left\langle c_{16} c_{40}\right\rangle \\
& +\left(a_{1}+a_{4}+a_{7}+a_{10}+a_{13}+a_{16}+a_{25}+a_{40}\right)\left\langle c_{37} c_{40}\right\rangle \\
& +\left(a_{1}+a_{4}+a_{7}+a_{10}+a_{13}+a_{16}+a_{25}+a_{37}+a_{40}\right)\left\langle c_{34} c_{37}\right\rangle \\
& +\left(a_{1}+a_{4}+a_{7}+a_{10}+a_{13}+a_{16}+a_{25}+a_{34}+a_{37}+a_{40}\right)\left\langle c_{31} c_{34}\right\rangle \\
& +\left(a_{1}+a_{4}+a_{7}+a_{10}+a_{13}+a_{16}+a_{25}+a_{31}+a_{34}+a_{37}+a_{40}\right)\left\langle c_{28} c_{31}\right\rangle \\
& +\left(-a_{14}\right)\left\langle c_{14} c_{15}\right\rangle+\left(-a_{14}-a_{15}\right)\left\langle c_{15} c_{41}\right\rangle+\left(a_{14}+a_{15}+a_{41}\right)\left\langle c_{36} c_{41}\right\rangle \\
& +\left(a_{14}+a_{15}+a_{36}+a_{41}\right)\left\langle c_{19} c_{36}\right\rangle+\left(a_{14}+a_{15}+a_{19}+a_{36}+a_{41}\right)\left\langle c_{9} c_{19}\right\rangle \\
& +\left(a_{9}+a_{14}+a_{15}+a_{19}+a_{36}+a_{41}\right)\left\langle c_{8} c_{9}\right\rangle \\
& +\left(-a_{8}-a_{9}-a_{14}-a_{15}-a_{19}-a_{36}-a_{41}\right)\left\langle c_{8} c_{20}\right\rangle \\
& +\left(-a_{8}-a_{9}-a_{14}-a_{15}-a_{19}-a_{20}-a_{36}-a_{41}\right)\left\langle c_{20} c_{35}\right\rangle \\
& +\left(a_{8}+a_{9}+a_{14}+a_{15}+a_{19}+a_{20}+a_{35}+a_{36}+a_{41}\right)\left\langle c_{30} c_{35}\right\rangle \\
& +\left(a_{8}+a_{9}+a_{14}+a_{15}+a_{19}+a_{20}+a_{30}+a_{35}+a_{36}+a_{41}\right)\left\langle c_{23} c_{30}\right\rangle \\
& +\left(a_{8}+a_{9}+a_{14}+a_{15}+a_{19}+a_{20}+a_{23}+a_{30}+a_{35}+a_{36}+a_{41}\right)\left\langle c_{3} c_{23}\right\rangle \\
& +\left(a_{3}+a_{8}+a_{9}+a_{14}+a_{15}+a_{19}+a_{20}+a_{23}+a_{30}+a_{35}+a_{36}+a_{41}\right)\left\langle c_{2} c_{3}\right\rangle \\
& +\left(-a_{2}-a_{3}-a_{8}-a_{9}-a_{14}-a_{15}-a_{19}-a_{20}-a_{23}-a_{30}-a_{35}-a_{36}\right. \\
& \left.-a_{41}\right)\left\langle c_{2} c_{24}\right\rangle+\left(-a_{2}-a_{3}-a_{8}-a_{9}-a_{14}-a_{15}-a_{19}-a_{20}-a_{23}-a_{24}-a_{30}\right. \\
& \left.-a_{35}-a_{36}-a_{41}\right)\left\langle c_{24} c_{29}\right\rangle+\left(a_{2}+a_{3}+a_{8}+a_{9}+a_{14}+a_{15}+a_{19}\right. \\
& \left.\left.+a_{20}+a_{23}+a_{24}+a_{29}+a_{30}+a_{35}+a_{36}+a_{41}\right)\left\langle c_{28} c_{29}\right\rangle\right)+\sum_{i=0}^{41} a_{i}\left\langle c_{28}\right\rangle .
\end{aligned}
$$

So $w_{0}$ is homologous to 0 -chain $\sum_{i=0}^{41} a_{i}\left\langle c_{28}\right\rangle$. Hence the 0 -cycle is homologous to an integral multiple of $\left\langle c_{28}\right\rangle$. Thus we get $H_{0}^{6}\left(M S S_{6} \sharp M S S_{6}\right) \cong \mathbb{Z}$.

From Example 2.12, Theorem 2.11, and the above, we have

$$
\begin{aligned}
-38=\chi\left(M S S_{6} \sharp M S S_{6}\right) & =\operatorname{rank} H_{0}^{6}\left(M S S_{6} \sharp M S S_{6}\right)-\operatorname{rank} H_{1}^{6}\left(M S S_{6} \sharp M S S_{6}\right) \\
& =1-\operatorname{rank} H_{1}^{6}\left(M S S_{6} \sharp M S S_{6}\right) .
\end{aligned}
$$

Therefore, $\operatorname{rank}_{1}^{6}\left(M S S_{6} \sharp M S S_{6}\right)=39$. It follows from Theorem 2.9 that $H_{1}^{6}\left(M S S_{6} \sharp M S S_{6}\right) \cong$ $\mathbb{Z}^{39}$.

\section{Acknowledgement}

The authors would like to express their sincere thanks to the referee for the valuable suggestions and comments which help to clarify the paper. 


\section{References}

[1] H. Arslan, I. Karaca, and A. Öztel, Homology groups of $n$-dimensional digital images, XXI. Turkish National Mathematics Symposium 2008, B1-13.

[2] R. Ayala, E. Domingues, A.R. Frances, A. Quintero, Digital homotopy with obstacles, Discrete Applied Mathematics 139(2004), 5-30.

[3] G. Bertrand, Simple points, topological numbers and geodesic neighborhoods in cubic grids, Pattern Recognition Letters 15(1994), 1003-1011.

[4] G. Bertrand and R. Malgouyres, Some topological properties of discrete surfaces, Journal of Mathematical Imaging and Vision 11(1999), 207-211.

[5] L. Boxer, Digitally continuous functions, Pattern Recognition Letters 15 (1994), 833-839.

[6] L. Boxer, A classical construction for the digital fundamental group, Journal of Mathematical Imaging and Vision 10(1999), 51-62.

[7] L. Boxer, Properties of digital homotopy, Journal of Mathematical Imaging and Vision 22 (2005), 19-26.

[8] L. Boxer, Homotopy properties of sphere-like digital images, Journal of Mathematical Imaging and Vision 24(2006), 167-175.

[9] L. Boxer, Digital products, wedges, and covering spaces, Journal of Mathematical Imaging and Vision 25 (2006), 169-171.

[10] L. Boxer, I. Karaca and A. Oztel, Topological invariants in Digital Images, Journal of Mathematical Sciences: Advances and Application, 11(2011) no 2, 109-140.

[11] L. Chen, Discrete surfaces and manifolds, Scientific Practical Computing, Rockville, MD, 2004.

[12] L. Chen and Y. Rong, Linear time recognition algorithms for topological invariants in 3D, Proceedings of International Conference on Pattern Recognition,2008.

[13] S.E. Han, Connected sum of digital closed surfaces, Information Sciences 176(2006), 332-348.

[14] S.E. Han, An extended digital $\left(k_{0}, k_{1}\right)$-continuity, Journal of Applied Mathematics and Computing 16(1-2)(2004), 445-452.

[15] G.T.Herman, Oriented surfaces in digital spaces, CVGIP: Graphical Models and Image Processing 55(1993), 381-396.

[16] T. Kacynski, K. Mischaikow, and M. Mrozek, Computational Homlogy, Springer-Verlag, New York, 2010.

[17] T.Y. Kong, A digital fundamental group, Computers and Graphics 13(1989), 159-166.

[18] T.Y. Kong and A. Rosenfeld, Digital topology - A brief introduction and bibliography, Topological Algorithms for the Digital Image Processing, Elsevier Science, Amsterdam, 1996.

[19] A. Rosenfeld, Digital topology, American Mathematical Monthly 86(1979), 76-87.

[20] A. Rosenfeld, 'Continuous' functions on digital pictures, Pattern Recognition Letters 4(1986), 177-184.

[21] Edwin H. Spanier, Algebraic Topology, Springer-Verlag, New York, 1966. 